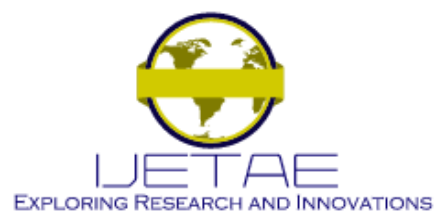

International Journal of Emerging Technology and Advanced Engineering

Website: www.ijetae.com (ISSN 2250-2459, ISO 9001:2008 Certified Journal, Volume 10, Issue 8, August 2020)

\title{
Parametric Continuously Programmable Infinite Impulse Response Filter for Non-Linear Real-Time DSP Applications
}

\author{
E. Elavarasi ${ }^{1}$, Dr. R. Manjunath ${ }^{2}$ \\ ${ }^{1}$ Research Scholar, ${ }^{2}$ Professor, AMCEC, Bangalore, India - 560083
}

\begin{abstract}
Considering the exponentially rise in non-linear signal filtering purposes, in this paper a novel parametric continuously programmable infinite impulse response (CPIIR) filter has been developed. Realizing the key upsurging significance of non-linear audio signal filtering, our proposed CPIIR filter model has been realized over parametric equalization system, which can have one or multiple IIR filters in cascade-design to perform continuous filtering. Structurally, our proposed model represents a PE solution containing shelving and second-order peaking CPIIR filters whose design parameters are optimized dynamically by reducing cost-function iteratively. Unlike classical approaches where merely filter coefficients are changed manually to cope up with non-linear signal filtering, our proposed parametric IIR filter employs sum-of-square error (SSE) as the costfunction to update filter design parameters like gain parameter, frequency and bandwidth. Noticeably, the use of SSE as cost-function intends to reduce error (i.e., difference between the target signal and the system frequency response) to optimize global gain parameter that eventually help updating other design parameter adaptively over different frequency-bins or windows. This process retains system response near 0 -dB line and thus maintains optimal filtering performance over swiftly varying signal response. Unlike major at hand meta-heuristic based search approaches for parameter tuning, our proposed model applies simple grid search concept that avoided significantly large computation and hardware requirements. MATLAB based simulation with Gaussian white noise embedded non-linear signals reveals that the proposed parametric CPIIR model can achieve optimal filtering performance and suitability towards hardware implementation.
\end{abstract}

Keywords-- Programmable Digital filter, Infinite Impulse Response filter, Parametric equalization, Sum-of- square error.

\section{INTRODUCTION}

The high-pace increase in digital signal processing (DSP) techniques have given rise to the different applications serving digital communication, signal-transmission systems, industrial communication, household purposes, internet of things (IoT), industrial monitoring and control, etc. Major at-hand applications undergo non-linear, abrupt changes in signal quality, signal alternation or manipulation because of the corrupt media, hardware-malfunction, corruptive channel conditions etc [1].
To ensure optimal signal reconstruction-quality different filtering approached have been proposed. Functionally, digital filters serve two key purposes; signal separation and reconstruction or restoration, where the earlier is considered when the signal is contaminated with interference or noise element(s). Unlike analog filters (AFs), digital filters (DFs) have performed better towards quality of signal construction and lower-computational complexity [1]. Though AFs are relatively low-cost, swift and have broadened amplitude/frequency range; it remains inferior over the DFs due to relatively superior performance and low hardware demands [1-3]. On the other hand, the recent development in DSP technique has broadened the use of DF over AFs [15]. Unlike classical AFs, DFs with dynamic programming assisted tuning can adapt non-linear behavioral model, which makes it more suitable across major DSP applications [5].

Though, DFs have broadened horizon towards different applications, increasing design complexity and higher filter's order demands significantly large hardware components and thus limits its uses [5][6]. Moreover, their ability as anti-aliasing filter too is limited; despite numerous researches have been towards the two key filter types; Finite Impulse Response (FIR) and Infinite Impulse Response (IIR) DFs [4]. Undeniably, FIR filters are relatively easier to design with low filter's order; however, factors like high hardware complexity, memory demand, and energy consumption limit its suitability for VLSI applications [1-5]. IIR DFs perform better than the FIR filters; though at the cost of increased design-cost [3][5]. Thus, low hardware utilization and eventual low-cost solution makes IIR superior over the FIR digital filters [5][7]. Structurally, the use of feedback component in IIR DF-design makes its transfer functions more complex than the FIR filter and hence demands optimal design parameter selection [8-10]. In major classical IIR filter designs the design parameters are manually assigned pre-test signal inputting; though it doesn't guarantee optimality of performance. On the other hand, being a feedback element-based filtering concept, it requires updating the design-parameters regularly to achieve fast and accurate signal reconstruction. To adapt timevarying, non-linear filtering demand continuous designparameter's update and tuning is must. 


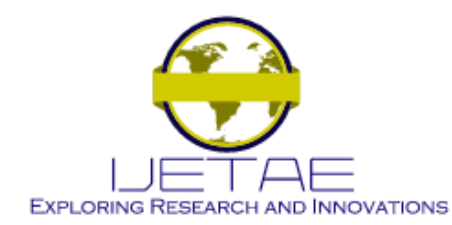

\section{International Journal of Emerging Technology and Advanced Engineering Website: www.ijetae.com (ISSN 2250-2459, ISO 9001:2008 Certified Journal, Volume 10, Issue 8, August 2020)}

The IIR filter model employing continuous designparameter update and tuning using certain mathematical approach or software-programming is called Continuously Programmable IIR filter (CPIIR). CPIIR can exploit error or the differences between input test signal impulse and the output impulse as a reference to update design-parameters iteratively. In major non-linear DSP components, filters are designed in such manner that its coefficients remain as the function of $\mathrm{H}(\mathrm{k})$ and $\mathrm{y}(\mathrm{k})$, signifying input test signal and the outputs signal, respectively. However, iterative errorsensitive coefficient estimation and update impose huge computational complexity, memory and energy exhaustion. Even-though IIR filters have superior significance towards DSP applications, very few researches could address CPIIR design, which can have superior prospect towards timevarying and non-linear signal filtering.

Considering the significance CPIIR filter towards nonlinear DSP applications, in this paper a novel parametric IIR filter design has been proposed which exploits SSE information signifying the difference between the output signal and the input signal impulses to estimate gain parameters and other design parameters, iteratively. Structurally, the proposed parametric CPIIR model represents a parametric equalization concept encompassing first-order shelving and second-order peaking IIR filters whose parameters are updated dynamically by reducing SSE to maintain $0-\mathrm{dB}$ line. The proposed filter model applies SSE as the cost-function to update filter design parameters like gain parameter, frequency and bandwidth. Noticeably, the use of SSE as cost-function intends to reduce error to optimize global gain parameter that eventually help updating other design parameter adaptively over different frequency-bins or windows. This process retains system response near $0-\mathrm{dB}$ line and thus maintains optimal filtering performance over swiftly varying signal response. The overall proposed model was developed using MATLAB2019b tool with SIMULINK toolbox. The proposed model was examined over the different input nonlinear, time-varying signals where it exhibited swift and accurate signal filtering, which affirms its suitability towards DSP components performing non-linear, timevarying signal filtering tasks.

\section{BACKGROUND}

Amongst the major DSP applications, DFs act as a linear time-invariant discrete system functional on the basis of the finite precision mechanisms.
Functionally, it applies a large number of recursive addition and multiplication functions [4], signifying a type of recursive system possessing fewer design parameters, lower memory, and eventually significantly low computational complexity than the traditional FIR digital filters. IIR DFs are defined in the form of linear constantcoefficient difference equation (1).

$$
y(n)=-\sum_{k=1}^{N} a_{k} y(n-k)+\sum_{k=0}^{M} b_{k} X(n-k)
$$

Performing $z$-transform of (1) to the equivalent $z$-domain function, this being a linear time-invariant discrete-time system can further be redefined as (2).

$$
H_{k}(z)=\frac{\sum_{k=0}^{M} b_{k} z^{-k}}{1+\sum_{k=1}^{N} a_{k} z^{-k}}
$$

Thus, based on (2), different kinds of IIR filter structures are derived. Though such designs differ from each other, they behave functionally equivalent. Typically, there are three key types of direct-form realizations; Direct-Form I, Direct-Form II and Transposed Direct-Form II architecture. Towards hardware utilization and complexity Direct-Form I structure demands a total of $M+N+1$ multiplication, $M+N$ additions, and $M+N+1$ memory locations. Similarly, the Direct-Form II design demands a total of $M+N+1$ multiplications, $M+N$ additions, and the $\{M, N\}$ memory locations. The Direct-Form II IIR design demands lower memory locations than the other architectures [4]. In this research we focus on enhancing Direct-Form II IIR design with CPIIR ability for PE based filtering purposes. The above defined differential equation gives the output signal as (3).

$$
\begin{aligned}
y[n]=\frac{1}{a_{0}} & \left(b_{0} x[n]+b_{1} x[n-1]+\cdots\right. \\
& +b_{p} x[n-p]-a_{1} y[n-1] \\
& -a_{2} y[n-2]-\cdots \\
& \left.-q_{Q} y[n-Q]\right)
\end{aligned}
$$

In (3), $P$ signifies the order of the feed-forward filter, $b_{i}$ represents the feedback filter order and $b_{i}$ represent the feedback filter coefficient. Here, $[n]$ presents the input signal, while the output signal is given by $y[n]$. Being a kind of recursive filter, its current output relies on previous outputs and therefore the difference equation can be redefined as (4) [5]. 


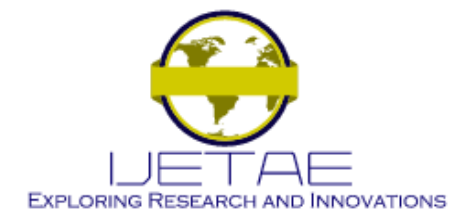

International Journal of Emerging Technology and Advanced Engineering Website: www.ijetae.com (ISSN 2250-2459, ISO 9001:2008 Certified Journal, Volume 10, Issue 8, August 2020)

$$
y[n]=\frac{1}{a_{0}}\left(\sum_{i=0}^{P} b_{i} x[n-i]-\sum_{j=1}^{Q} a_{j} y[n-j]\right)
$$

direct output for the same can be obtained as (7).

$$
H(z)=C+\sum_{k=1}^{K} \frac{A_{k}}{1-p_{k} z^{-1}}
$$

The target is obtained by means of the mathematical factoring (say, cascading 2nd order sub-blocks) over $H(z)$, and thus the overall transfer function is defined as (8).

$$
H(z)=\prod_{k=1}^{K} H_{k}(z)
$$

In (7), $K$ states the integer part of $\left(\frac{N+1}{2}\right)$, while $H_{k}(z)$ possesses $2^{\text {nd }}$ order form (shelving filter, Section V) (9).

$$
H_{k}(z)=\frac{b_{k 0}+b_{k 1} z^{-1}+b_{k 2} z^{-2}}{1+a_{k 1} z^{-1}+a_{k 2} z^{-2}}
$$

The mechanism of grouping the pair of complexconjugate poles and the pair of complex-conjugate zeros becomes complex. The result of a quantized IIR filter primarily rely on the sequential ordering of the $K$ subblocks in addition to the method in which both numerator as well as denominator parts are paired together. Observing major components and corresponding constraints, it can be inferred that in addition to the coefficient optimization, selection of filter design, low-order filter selection and allied design parameter (i.e., frequency, gain, bandwidth) selection can be vital to retain optimal non-linear filtering target. Considering it as motivation, we reviewed a few related literatures, whose snippets are given in the subsequent section.

\section{RELATED WORK}

Exploring major literatures, it can be inferred that despite of a limited researches towards IIR filter design optimization, majority of the existing solutions perform limited due to arbitrary design parameter selection. Such designs results into ripple and error in the output signal which affects overall signal quality. Considering meta heuristic based design parameter optimization methods [2231], though these methods play vital role towards square error or ripples suppression within passband and stopband, however at the cost of high computational cost, delay etc. It might even increase memory and area consumption making them unsuitable for VLSI or FPGA implementation.

\section{PROBLEM FORMULATION}

Enabling non-linear signal filtering requires adapting optimal or suitable DF design parameter(s) selection. Considering a case of real-world audio signal filtering purpose such as load-speaker signal equalization, PE technique has been applauded across academia-corporate, though the soul of such approaches exists within the use of a suitable IIR filter design. Enhancing IIR in response to the impulse error can enable transient and more efficient non-linear filtering. In PE, IIR filters are applied in a cascade manner where these filters can be of different types like shelving or peaking filter structure. Its architecture makes it suitable for low order filter design and its hardware/VLSI implementation [32]. In major IIR based PE solutions adjusting design parameters such as central frequency, bandwidth, and gain helps retaining optimal filter-performance. Unlike manual design parameter assignment, an automatic parameter estimation, selection and tuning can be more efficient to achieve $0-\mathrm{dB}$ line performance. Though, the majority of the PE solutions encompass multiple IIR filter (it can be the type of shelving or peaking or their cascade design) [32-34], we focus more on enhancing IIR design which could yield optimal performance even with lower order and suitable gain. We consider that the enhanced and CPIIR implementation of second order peaking filter with SSE as cost-function can help optimizing global gain value of the IIR filter. The proposed model optimises design parameters in such manner that it retains $0-\mathrm{dB}$ line, with reference to which the global gain parameter (GGP) is estimated iteratively and updated to the CPIIR of the PE-filter. The proposed model functions in such manner that it optimises filter design parameters including gain, frequency and bandwidth while reducing the cost function (near $0-\mathrm{dB}$ line) to retain optimal filter performance over non-linear input signal. Structurally, being cascaded CPIIR architecture for PE, to adopt dynamic update our proposed model inherits the characteristics of the first and the second order peaking and shelving digital filters, respectively. It yields low-order PEfilter for non-linear data (containing mixed noise components) filtering. This approach intends to retain better magnitude equalization with no impact on phase response. Here, reducing cost-function states the compensation of the error to retain target response by equalizing energetic frequency peaks over dip. Our proposed adaptive filtering concept enables inclusion of the gradient based compensation enabled by means of adaptive global gain update (in reference to the 0 - $\mathrm{dB}$ line). In our proposed model, least square method (LS) has been applied in closed loop to estimate the global gain parameter. 


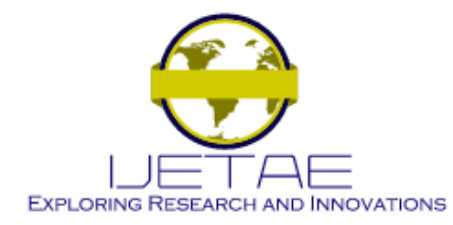

\section{International Journal of Emerging Technology and Advanced Engineering Website: www.ijetae.com (ISSN 2250-2459, ISO 9001:2008 Certified Journal, Volume 10, Issue 8, August 2020)}

Unlike major at hand solutions like meta-heuristic algorithms, the propose method applies simple grid-search concept which enables other design parameter (frequency and bandwidth) estimation more efficient, while supporting its suitability towards hardware implementation. The detailed discussion of the proposed parametric CPIIR model is given in the subsequent sections.

\section{SYSTEM MODEL}

Let $H_{0}(k)$ be the frequency response to be filtered. Here, $k$ states the radial frequency index characterising the transfer function for each frequency bin and $f_{s}$ be the sampling frequency while the filtered frequency response be $H_{s}(k)$ with $s$ CPIIR filters. As already stated, the parametric CPIIR filter can be applied in a cascaded $s$-filter design where each IIR filter can be either peaking filter (with filter order $\mathrm{m}=2$ ) or the shelving filter of order $(m=1)$. Our proposed filter design functions as non-linearin-the-gain (NLIG) form which is applied with a negative gain to retrieve a cut-within the frequency response. In major digital filtering concepts, the focus is made on reducing the fluctuations between the system response $H_{0}(k)$ and the target response $T_{k}$ using filter(s) of $M$ order, where the complete response used to be $F_{M}(k)$. It intends to equalizes the responses by means of an error function (10).

$$
E_{M}(k)=W(k)\left\{H_{0}(k) \cdot F_{M}(k)-T(k)\right\}
$$

In (10), $W(k)$ states an adaptive weight function which is assigned to provide adaptive (more or less) importance towards the response error (here., SSE) at certain frequency bins. Noticeably, spectral peaks can also be applied as the cost-function to perform error minimization using (11). This approach (11) applied error value on linear scale than the logarithmic scale.

$$
\epsilon_{M}^{l i n}=\frac{1}{N} \sum_{k}\left|W(k)\left\{H_{0}(k) \cdot F_{M}(k)-T(k)\right\}\right|
$$

Unlike classical approaches (10) for cost-estimation the model derived in (12) estimates cost-function on a linear scale that gives emphasis on those specific sections which requires to be equalized for corresponding magnitude response above the target. It helps removing peak impulse above the target frequency response. For better and transient filtering, both areas of the magnitude above as well as below the target response can be taken into consideration.
We inherit this concept to enable swift non-linear filtering where the largest section of the frequency response turns out to be the one for filtering while the half-way point existing in between the two zero-crossing points and the negation of its level (in $\mathrm{dB}$ ) characterises the central frequency and filter-gain (or Q-value), respectively. Here, we assume that the filter response embodies both peaks as well as dips above and below the expected target response, correspondingly. We consider applies $0-\mathrm{dB}$ line aspect which makes filter response more accurate [34]. Unlike classical works such as [34] where authors applied predefined value of bandwidth, our proposed model estimates these design parameters (i.e., cut-off frequency, bandwidth and gain Q-value) dynamically over input signal (while reducing SSE cost-function. In major existing solutions authors have applied meta-heuristic approaches such as GA, ACO, PSO etc for parameter estimation; however, their computationally exhaustive and timeconsuming nature can't be ignored. Though, gradient-based parameter enhancement by means of the numerator and the denominator coefficient update can be done; however, it functions irrespective of the design parameters such as gain parameter, bandwidth and frequency [35]. Summarily, our proposed parametric CPIIR model applies SSE information to update the aforesaid design parameters. A brief of the SSE cost-function estimation and PE using CPIIR model is given in the subsequent section.

\section{A. SSE based Filtering and Equalization}

Our proposed model defined (12) as the fundamental SSE model for cost-function definition towards non-linear filtering.

$$
\epsilon_{M}^{S S E}=\frac{1}{N} \sum_{L}\left(W(k)\left[H_{0}(k) \cdot F_{M}(k)-T(k)\right]\right)
$$

This method enables better error traceability for transient filtering and equalization due to continuous gradient update and tuning. For line-in-gain (LIG) form, it provides closed loop model for global gain estimation and enables automatic parameter update. Unlike classical methods with fixed initial parameters, our proposed approach tunes initial value dynamically to retain target performance with nearoptimal design values. It also helps estimating global gain parameter dynamically in a closed form (in reference to the SSE value per frequency-bin). This method emphasizes on error-suppression by considering perceptually relevant frequency peaks in comparison to the dips information. 


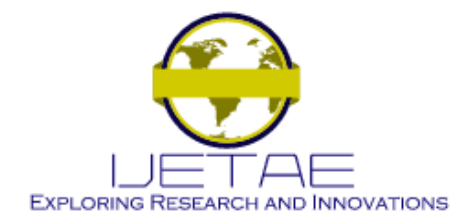

\section{International Journal of Emerging Technology and Advanced Engineering Website: www.ijetae.com (ISSN 2250-2459, ISO 9001:2008 Certified Journal, Volume 10, Issue 8, August 2020)}

In the proposed design represents parametric CPIIR filter solution with the minimum-phase response that characterises its frequency response $H(\omega)$ to be estimated by means of corresponding magnitude response. Mathematically, $\emptyset_{H}(\omega)$ is obtained by applying inverse Hilbert transform $\mathcal{H}^{-1}()=.-\mathcal{H}($.$) . In other words,$

$$
\begin{aligned}
H(\omega) & =\left|H(\omega) e^{j \varnothing H(\omega)}\right| \\
\emptyset_{H}(\omega) & =-\mathcal{H}\{\ln |H(\omega)|\}
\end{aligned}
$$

In frequency domain,

$$
\ln H(\omega)=\ln \left|H(\omega)+j \emptyset_{H}(\omega)\right|
$$

In case of digital domain, the phase response of the minimum phase frequency response $H(k)$ is estimated as the imaginary component of the discrete Fourier transform of the real periodic cepstrum. Mathematically,

$$
\emptyset_{H}(k)=I\{D F T\{\operatorname{fold}\{\hat{h}(n)\}\}\}
$$

In (15) $\hat{h}(n)$ presents the DFT of the folded real periodic cepstrum and hence with the associations existing in between the magnitude and the phase of a minimum-phase frequency (13), reducing the cost function (12) can achieve filtering target. Thus, with above decided SSE cost function we performed linear-in-the-gain (LIG) parametric IIR filter design.

\section{B. LIG CPIIR Filter}

Typically, majority of the cascaded IIR based PE solutions embody first- and second-order IIR filters, while constraining filter magnitude response which is often defined at the zero frequency, Nyquist frequency, central frequency for peaking filters. Though, there can be different approaches for parameterization while satisfying defined constraints; the concept used with the first and the second-order IIR is more effective [36]. With this motive, we applied PE design with CPIIR comprising all-pass (AP) filter $A_{m}(z)$ of order $m$ in a feed-forward path. For the allpass filtering to be independent of the gain $V$, the CPIIR to be applied possesses the transfer-function given in (16).

$$
\begin{gathered}
F_{m}(z)=\frac{1}{2}\left[(1+V)+(1-V) A_{m}(z)\right] \\
=\frac{1}{2}\left[\left(1+A_{m}(z)\right)+V\left(1-A_{m}(z)\right)\right]
\end{gathered}
$$

Noticeably, for $V>0$ the filter response would be of minimum-phase type while for $V<0$ it would possess the maximum phase and therefore in this paper we considered IIR filter with only positive linear gain.
Our proposed model can be hypothesized to be energysaving as the energy of the output signal of the AP filter remains equivalent to the energy of its input signal and therefore $y^{n}(n)=x(n)+z(n)$ in conjunction with a notch and $y^{\beta}(n)=x(n)-z(\mathrm{n})$ (in reference to the resonance) remains orthogonal. This criterion signifies that when $V$ doesn't exist in the AP filter transfer function, it operates on the resonant response $y^{\beta}(n)$. On the contrary, the notch response $y^{n}(n)$ remains unchanged even with changed value of the gain $V$. Our proposed model with LIG implementation model is capable of producing both boost as well as cut in the response, irrespective of the fact that the cut response intends to consume minimized bandwidth [36].

\section{First-order shelving filters}

In our proposed parametric CPIIR model, we apply firstorder shelving filter that acts once the lowest or maximum fraction of the system frequency response requires to be either enhanced or minimized. This filter is characterised in the form of the gain parameter $V$ and the transition frequency $f_{c}$. Thus, applying the filter architecture as derived in (16-17), we obtained a first-order shelving filter as both low as well as high frequency. Mathematically,

$$
\begin{aligned}
& A_{1}^{L F}(z)=\frac{a_{L F}-z^{-1}}{1-a_{L F} z^{-1}} \\
& A_{1}^{H F}(z)=\frac{a_{H F}+z^{-1}}{1+a_{H F} z^{-1}}
\end{aligned}
$$

The LIG form can be retrieved in terms of the parameters $f_{c}$ and the sampling frequency $f_{s}$.

$$
\begin{aligned}
a_{L F}^{b} & =\frac{1-\tan \left(\pi f_{c} / f_{s}\right)}{1+\tan \left(\pi f_{c} / f_{s}\right)} \\
a_{H F}^{b} & =\frac{\tan \left(\pi f_{c} / f_{s}\right)-1}{\tan \left(\pi f_{c} / f_{s}\right)+1}
\end{aligned}
$$

The above equation reveals that the all-pass filter doesn't rely on the gain parameter $V$, nevertheless in case of $0<V<1$, when the filter signifies a cut, the transition frequency of the filter response intends to incline towards the lower frequencies (in case of high-pass filter it inclines towards the higher frequencies). To retrieve a cut response, especially when $0<V<1$ for LIG, the gain parameter $V$ is substituted by 1 , which results into (22) and (23).

$$
\begin{aligned}
& a_{L F}^{C}=\frac{V-\tan \left(\pi f_{c} / f_{s}\right)}{V+\tan \left(\pi f_{c} / f_{s}\right)} \\
& a_{H F}^{c}=\frac{\tan \left(\pi f_{c} / f_{s}\right)-V}{\tan \left(\pi f_{c} / f_{s}\right)+V}
\end{aligned}
$$




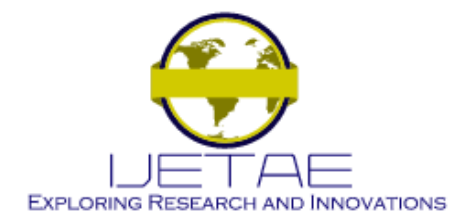

\section{International Journal of Emerging Technology and Advanced Engineering Website: www.ijetae.com (ISSN 2250-2459, ISO 9001:2008 Certified Journal, Volume 10, Issue 8, August 2020)}

The above derived model (22-23) results into a shelving filter model, which can further be redefined to give simple approach to boost the response with gain $V$ [36-38]. Eventually, the consequent IIR filter architecture represents a nonlinear in the gain (NLIG) parameter.

\section{Second order Peaking IIR filter}

Peaking IIR filters are applied to compensate peaks and dips of the system (frequency) response. Considering above derived function of IIR filter with coefficients (9), the peaking IIR filter can be derived in the form of secondorder all-pass filter (24).

$$
A_{2}(z)=\frac{a+d(1+a) z^{-1}+z^{-2}}{1+d(1+a) z^{-1}+a z^{-2}}
$$

In (24), $d=-\cos \left(2 \pi f_{0} / f_{s}\right), f_{0}$ signifies the central frequency of the $2^{\text {nd }}$ order peaking IIR filter. Now, the LIG form can be retrieved in terms of the bandwidth parameter $a(25)$.

$$
a^{b}=\frac{\tan \left(\pi f_{b} / f_{s}\right)-1}{\tan \left(\pi f_{b} / f_{s}\right)+1}
$$

In (25), $f_{b}$ states the $-3-\mathrm{dB}$ notch bandwidth retrieved conditioned at $V=0$. To achieve symmetric boost and cut responses dynamically (conditioned at $0<V<1$ ), the following is applied [36-38].

$$
a^{b}=\frac{\tan \left(\pi f_{b} / f_{s}\right)-V}{\tan \left(\pi f_{b} / f_{s}\right)+V}
$$

In above derived model the linear dependency in reference to the gain parameter is lost and therefore we considered only LIG form to achieve automatic parametric tuning and dynamic equalisation over non-linear input signal. Though, in both NLIG and LIG form shelving or peaking filters can be applied to obtain and update filter parameters dynamically. One of the key significances of applying LIG form is its ability to exhibit orthogonality as well as linearity that enables a closed-form solution for dynamic gain parameter estimation (which eventually helps retaining $0-\mathrm{dB}$ line). In our proposed model, we redefine the cost-function as (27).

$$
\begin{aligned}
d z S Z \quad \epsilon_{m}^{S S E}= & \frac{1}{N} \sum_{k}\left(W ( k ) \left\{\frac { 1 } { 2 } H _ { 0 } ( k ) \left[F_{m}^{\eta}(k)\right.\right.\right. \\
& \left.\left.\left.+V F_{m}^{\beta}(k)\right]-T(k)\right\}\right)^{2}
\end{aligned}
$$

where, $($ for $\mathrm{k}=1,2 \ldots \mathrm{N})$

$$
\begin{aligned}
& F_{m}^{\eta}(k)=1+A_{m}(k) \\
& F_{m}^{\beta}(k)=1-A_{m}(k)
\end{aligned}
$$

To achieve cost-function minimization we assigned the first order partial derive of (27) as zero (with respect to the gain parameter $V$ ).
Here, the gain parameter is obtained using (29).

$$
\widehat{V}=\frac{\sum_{k}|W(k)|^{2} F_{m}^{\beta *}(k) H_{0}^{*}(k) T(k)}{\sum_{k}|W(k)|^{2}\left|H_{0}(k)\right|^{2}\left|F_{m}^{\beta *}(k)\right|^{2}}
$$

In above derived function $\{.\}^{*}$ states the complex conjugation which doesn't depend on $F_{m}^{\eta}(k)$ due to the orthogonality in between $F_{m}^{\eta}(k)$ and $F_{m}^{\beta}(k)$. In our proposed approach this ability is being applied to perform signal equalization where selecting optimal value of the gain parameter at certain instant it equalizes the signal of that corresponding frequency bin. Once obtaining the optimal gain parameter as discussed above the CPIIR is tuned with the optimal gain $\widehat{V}_{s}$ of the s-th IIR filter, which is done by replacing frequency response $H_{o}(z)$ by $H_{s-1}(z)$.

\section{E. System Implementation}

In our proposed parametric CPIIR DF model, we designed a non-linear filter structure of $M$-order containing s-cascaded (IIR) filters, nevertheless a PE filter can be designed with filter order $m_{s}=1$ (shelving filter) or $m_{s}=2$ (peaking). With such design, the filter's frequency response can be modelled as (30).

$$
F_{m}(k)=C \prod_{s=1}^{s} F_{m, s}(k) \text { with } M=\sum_{s=1}^{s} m_{s}
$$

In (30), $s$ refers the filter operating while $C$ being the global gain value. In our proposed CPIIR filter the design parameters are optimized dynamically while minimizing the cost-function $\mathrm{F}\left(a_{s}, d_{s}, V_{s}\right)$. Mathematically, (31).

$\epsilon_{s, m_{s}}^{S S E}=\frac{1}{N} \sum_{k}\left(W(k)\left\{H_{s-1}(k) F_{m s}(k)-T(k)\right\}\right)^{2}$

As stated in (31), with $H_{s-1}$ the CPIIR filter response comprises the previous $s-1$ filtering components. When executing the model, our proposed approach automatically defines the target response $T(k)$ and the input system response (input signal with the white noise) $H_{0}(k)$. In our proposed model, the global gain parameter $(\mathrm{C}$, in 30$)$ is obtained in a closed form. Subsequently, the proposed model assigns the initial design parameters followed by tuning of the optimal gain parameter to help retrieving the central frequency and bandwidth values. We apply a computationally efficient grid search optimization concept which updates the initial parameters and gain values, distinctly. Here, the global gain parameters are optimized continuously while retaining $0-\mathrm{dB}$ line. In other words, our proposed model retains the global gain value optimal by setting the system magnitude response centred near $0-\mathrm{dB}$ line of the target frequency response. 


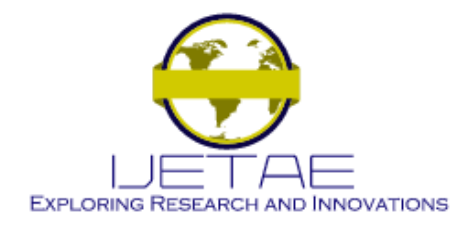

\section{International Journal of Emerging Technology and Advanced Engineering Website: www.ijetae.com (ISSN 2250-2459, ISO 9001:2008 Certified Journal, Volume 10, Issue 8, August 2020)}

Though the proposed model can also perform satisfactory by maintaining $0-\mathrm{dB}$ line (by visual analysis or inspection) or maintaining average of the system response within the target frequency response (say, mid frequencies); however, it can't guarantee optimality of the solution. On the contrary, SSE cost-function minimization can yield optimal performance without imposing higherorder computational cost. The global gain parameter has been obtained as per (32).

$$
\hat{C}=\frac{\sum_{k}|W(k)|^{2} H_{0}^{*}(k) T(k)}{\sum_{k}|W(k)|^{2}\left|H_{0}(k)\right|^{2}}
$$

The global gain parameter $C$ is stated to be a scaling factor which intends to maintain the system response near the targeted $0-\mathrm{dB}$ line by minimizing the corresponding cost-function. Realizing the fact that the major SSE costfunction minimization concepts emphasize on the peaks of the system magnitude response that make it incline to have dips which are more prominent in comparison to the peaks in reference to the targeted frequency response. It can cause biased boosting of the dips and hence can make system inefficient over exceedingly fast non-linear input. Considering this fact, we suggest applying an offset of certain small (can be decided based on the DSP applications) $\mathrm{dB}$ to the global gain value $C$. It can ensure maintaining optimal filtering by equalizing peaks over dips to restore $0-\mathrm{dB}$ line. We applied a simple linear search model for frequency and bandwidth update. In this method, we defined a pole grid where the radius and the angle of the complex poles defined the bandwidth $f_{b}$ and the central frequency $f_{0}$ of the second-order peaking CPIIR filter. The radius of the real-poles characterises the transition frequencies $f_{c}$ of the los-pass filter (i.e., positive real poles) and the high-pass filter (negative real poles) shelving filter. The gain of the filter design is achieved as discussed above by reducing SSE cost-function. Thus, with the updated values of the design parameters the proposed CPIIR filter equalizes the output signal to retain target response. Considering hardware suitability and limited specifications we defined minimum value of the gain parameter $V_{\min }$ as $0.25 \mathrm{~dB}$ while the maximum was fixed as $V_{\max }=4$. Noticeably, to maintain IIR sanity and principle which expects system response to be of minimum phase form [36], our proposed model maintains global gain $V$ as realpositive value.

\section{RESUlTS AND DISCUSSION}

In this paper the key focus was made on designing an efficient continuously programmable or adaptive IIR filter design to cope up with the non-linear DSP purposes or the signal(s) filtering.
Unlike FIR filter, IIR does have lower complexity of design; however, relatively large hardware utiliation and allied complexity, which is primarily introduced because of the feedback-type design. Despite such fact, IIR digital filters have been applied in greater extent than the classical FIR filters. One of the key applications of the IIR DFs is in audio systems, where it is applied as supplimentary filter to enhance signal level and supress noise or interfrence components. Most of the audio system these days employ parameteric equalization concept to denoise the signal, where the PE concept itself applies IIR filter with feedback loop architecture. Considering this as target application we designed PE solution with continuously programmable IIR filter design. Here, the key purpose of applying CPIIR as the PE filter component was its ability to adop non-linear filtering without imposing large hardware cost or complexity. Moreover, to enhance overall design, especially to cope up with non-linear input filtering, we optimized IIR filter with CPDF feature where the key design parameters inlcuding cut-off frequency, bandwidth, and gain parameters were enhanced iteratively. Though, another approach to optimise IIR in terms of CPIIR could be to optimise filter coefficients (nominator and denominator of (9)), it could be exhaustive and could limit applicability towards hardware implementation or CMOS purpose. Realizing this fact, we cosnidered SSE sensitive design parameter estimation and tuning, especially for gain-parameter, cut-off frequency and bandwidth. Noticeably, our proposed CPIIR based PE solution encompassed first-order Shelving IIR filter cascaded with second-order Peaking IIR filter. In classical PE filter designs where at first the parameter(s) of the shelving filter is obtained and tuned, which is followed by peaking filter tuning and subsquently filtering is performed. Realizing the need of low-order filter for better hardware suitability, we applied second order filter (i.e., the number of filter order as two). Here, in PE design we focused on enhancing second order peaking CPIIR filter by estimating its design parameter iteratively in refernce to the SSE as objective or cost-function, which was estimated as the difference between the target signal and the system frequency response. The CPIIR filters were optimized over simulation period, where to estimate gain parameters iteratively we applied SSE as cost-function. To optimise the filter design parameters, we hypothesized $0-\mathrm{dB}$ line as reference where reducing SSE the global gain parameter was obtained iteratively for each bin or window.

To ensure fair comparison of performance, we considered same target response for a parallel low-pass Butterworth filter, where the input range for equalization was defined in the range of $1 \mathrm{kHz}$ and $45 \mathrm{kHz}$. 


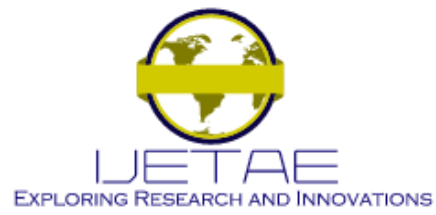

\section{International Journal of Emerging Technology and Advanced Engineering Website: www.ijetae.com (ISSN 2250-2459, ISO 9001:2008 Certified Journal, Volume 10, Issue 8, August 2020)}

We built the target response in such manner that it matches the band-pass characteristics using second-order high-pass and low pass Butterworth filters with the centre frequency of $1000 \mathrm{~Hz}$. The response was scaled in such manner that the $0-\mathrm{dB}$ line of the target impulse response remains in synched with the response mean value, which fulfils the need of the peaks and dips to be equalized. We applied the same termination condition for reference filter, which was considered as SSE reduction (in the number of previous iterations is lower than the tolerance $10^{-8}$ or the maximum allowed ripple (in $\mathrm{dB}$ ). Here, we assigned initial Q-value as Romas et al [34][39] suggestions. We assigned initial Q-value as 4.5, while the initial gain was assigned as $12 \mathrm{~dB}$. To be noted, the input signal was added with white noise which was expected to be supressed over the simulation to realize real-world audio-system such as loudspeaker. We examined the performance of the proposed CPIIR assisted PE filter design using spectrogram simulation where the simulation results were obtained in terms of filter's magnitude response $(\mathrm{dB})$, phase response $(\mathrm{dB})$, group-delay response (s), complementary cumulative distribution function (CCDF) performance, and distortion. Here, the start of frequency span was fixed as $20 \mathrm{~Hz}$, where the stop-frequency was set as $25 \mathrm{kHz}$. The attenuation was $60 \mathrm{~dB}$ over signal being processed over continuous windows. Additionally, the sample rate was fixed as 45 $\mathrm{kHz}$, and we applied Kaiser window as an approximator to the prolate spheroidal window (say, window (approximation) function for spectral analysis).

We obtained filter responses over the frequency range of (mean value) $400 \mathrm{~Hz}$ to $22.5 \mathrm{KHz}$. Since the filter output is a complex system with both magnitude as well as phase, we examined these output in terms of magnitude and phase response, in addition to the group delay response. Typically, the magnitude response of the filter is characterised in terms of the frequency bands the filter passes or rejects. Fig. 1 presents the frequency magnitude response by the proposed PE CPIIR filter. Observing the results, it can be found that the proposed CPDF model converges soon over the frequency span. Here, the pass band of the filter exists in between $400 \mathrm{~Hz}$ to almost $3 \mathrm{KHz}$ (cut-off frequency), while the stop band starts from $3 \mathrm{KHz}$. Noticeably, the proposed PE CPIIR filter, similar to the low-pass Butterworth filter doesn't have any ripple and therefore the amplitude monotonically decreases (Fig. 1). Since, the result (Y-axis of Fig. 2) is in $\mathrm{dB}$, the magnitude signifies the ratio of the output to the input magnitude. Similarly, the phase response of the proposed filter is given in Fig. 2. Fig. 2 represents the radian phase shift added to the phase of each sinusoidal component of the input signal.
Fig. 3 presents the group delay caused by the proposed CPIIR assisted PE model. Noticeably, our proposed filter designs achieve swift convergence and thus dhows negligible delay. Recalling the fact that the phase delay states the time-delay (in seconds) experienced by each sinusoidal component of the input signal. The simulation result states (Fig. 3) that the proposed filter model achieves significantly low delay. Mathematically, group delay is defined as (33).

$$
D(\omega) \triangleq-\frac{d}{d \omega} \Theta(\omega)
$$

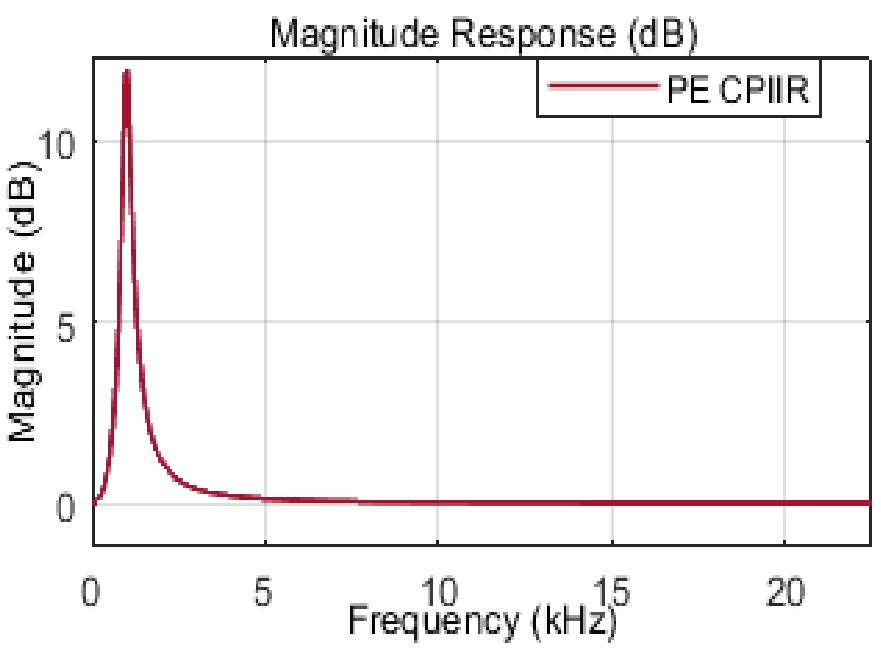

Fig. 1 Magnitude Response (dB)

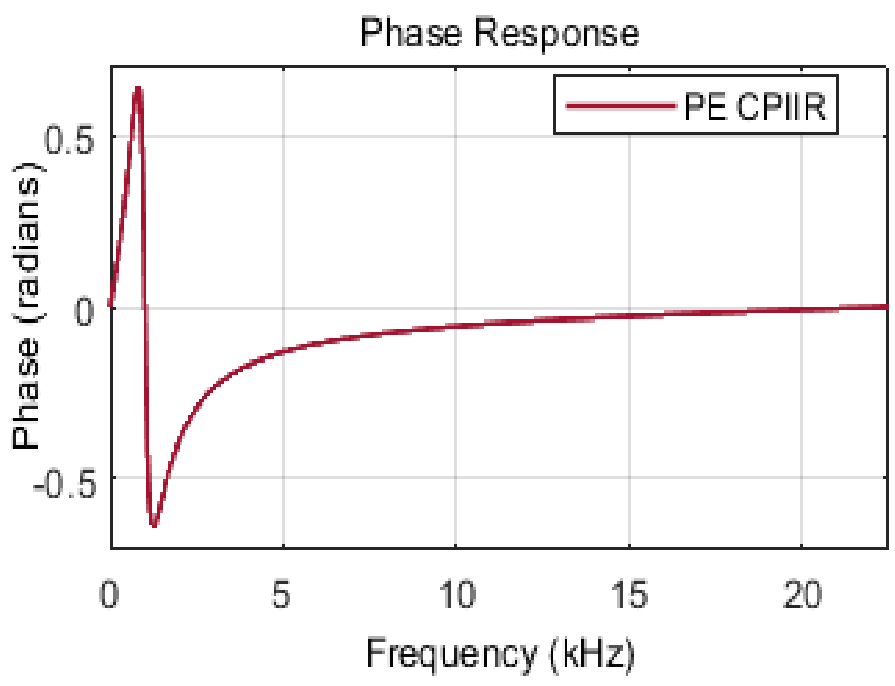

Fig. 2 Phase Response (radians) 


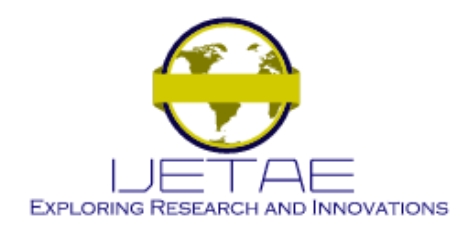

International Journal of Emerging Technology and Advanced Engineering

Website: www.ijetae.com (ISSN 2250-2459, ISO 9001:2008 Certified Journal, Volume 10, Issue 8, August 2020)

Typically for a linear phase response $\Theta(\omega)=-\alpha \omega$, with $\alpha$ as constant, both group as well as phase delay used to be equivalent, and hence can be called as time-delay (with $\alpha$ samples). On contrary, for non-linear phase response, the relative phases of the sinusoidal signal undergo alteration causing certain percussive sound or smearing. In this reference, observing the group delay performance by the proposed filter design (Fig. 3), it can be found that the proposed design retains zero-delay in most cases. It affirms robustness of the proposed filter to avoid smearing or percussive kind of alteration over fast nonlinear signal filtering.

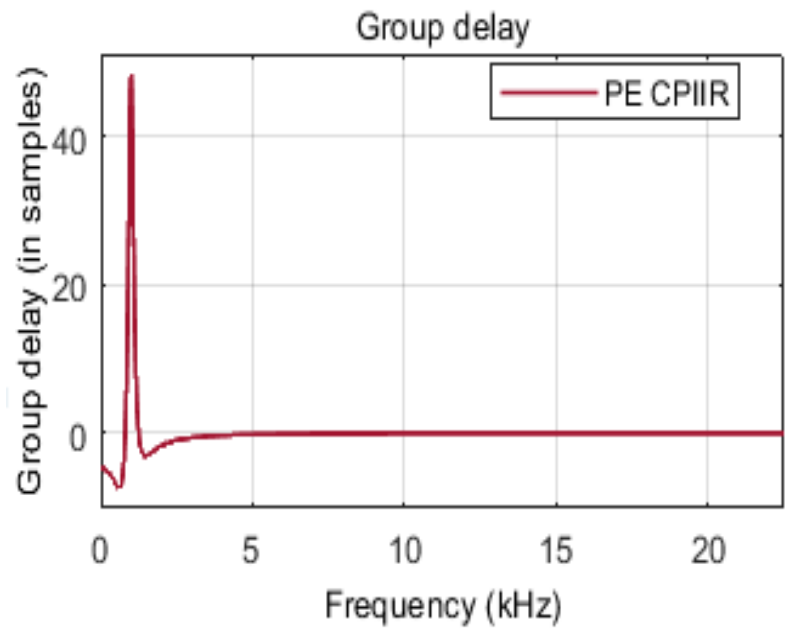

Fig. 3 Group delay performance

Fig. 4 presents the CCDF performance signifying dB over average power and quantification probability. The proposed system achieved targeted response without any randomness or inconsistence. In addition, the result (Fig. 4) exhibits how much time the response signal spends at or above a pre-defined target power level. Here, the power level is defined in terms of $\mathrm{dB}$ in reference to the average power. Here, each line presenting red, blue and green colour line shows a specific power level above the average (Fig. 4(b)).

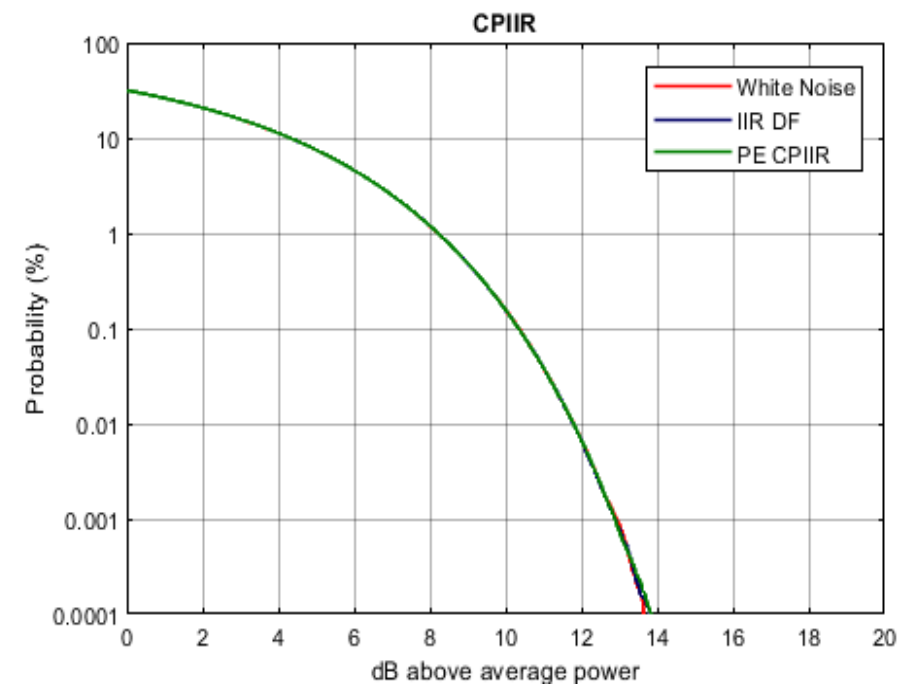

Fig 4(a) CCDF performance (power level vs probability)

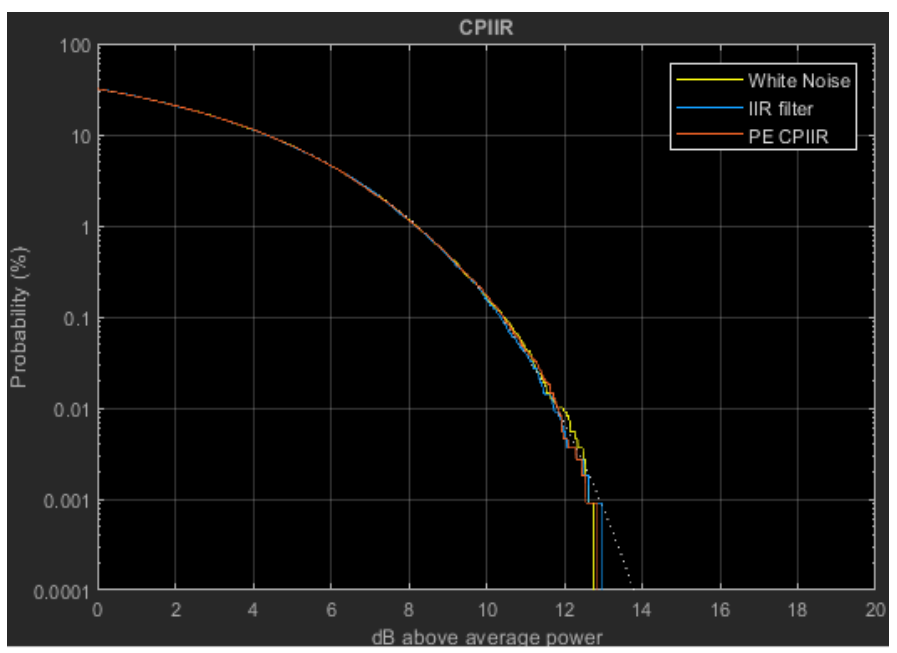

Fig. 4(b) CCDF Performance with reference to the Gaussian reference line

The fraction of time the impulse response signal spends at or below the Band-limited Gaussian noise CCDF reference line (dotted line in Fig. 4(b)) characterises the probability for that specific power level (x-axis). 


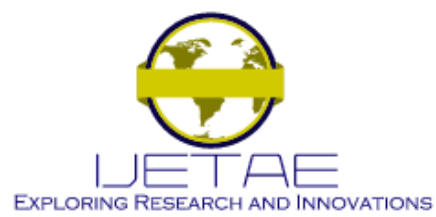

International Journal of Emerging Technology and Advanced Engineering

Website: www.ijetae.com (ISSN 2250-2459, ISO 9001:2008 Certified Journal, Volume 10, Issue 8, August 2020)

The results affirm expected performance with very small percentile of the probability that the target response remains below the reference line. In other words, the filter response remains always on the top of the reference line signifying satisfactory performance. Fig. 5 presents the filtering visualization of the proposed PE CPIIR filter where it can be found that the proposed design retains optimal filtering performance in synch with the target response. The power spectrum analysis using spectrogram of the proposed PE CPIIR filter design is given in Fig. 6.

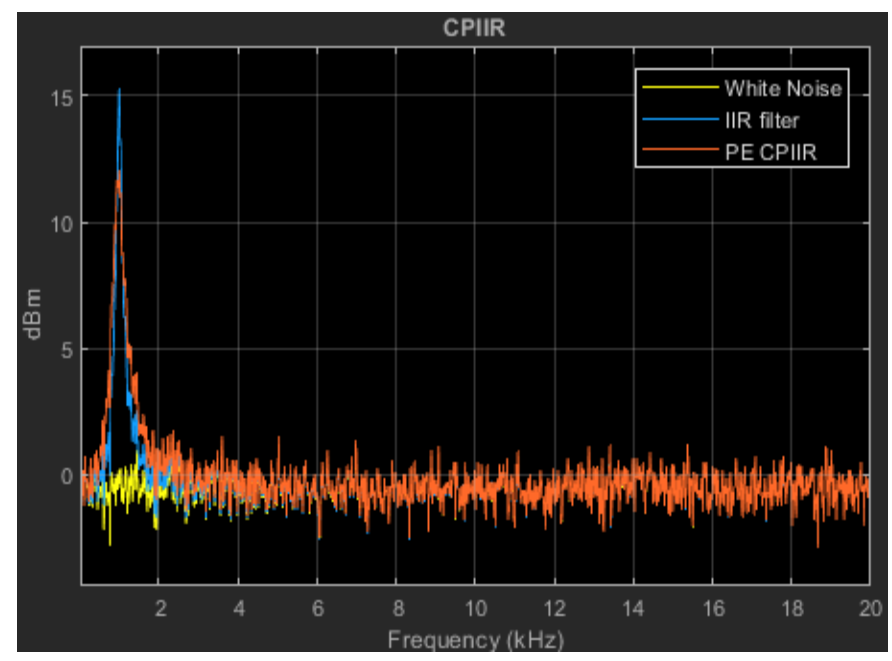

Fig. 5 Filtering performance over varying frequency windows

In addition to the above discussed filter response assessment, we analysed the performance in terms of the (signal or response impulse) distortion. We measured signal distortion at the frequency of $8.11 \mathrm{kHz}$, which shows $0.85 \mathrm{~dB}$ of signal distortion, while another measurement at the frequency of $16.2 \mathrm{kHz}$ showed $-0.91 \mathrm{~dB}$ of distortion. Undeniably, the distortion observed was small enough to cause any significant impact on the output response quality. Additionally, over aforesaid distortion assessment period, we observed SNR and SINAD of $22.88 \mathrm{dBc}$, while SFDR was measured as $0.02 \mathrm{dBc}$. These results affirm suitability of the proposed CPIIR filter towards non-linear signal filtering.

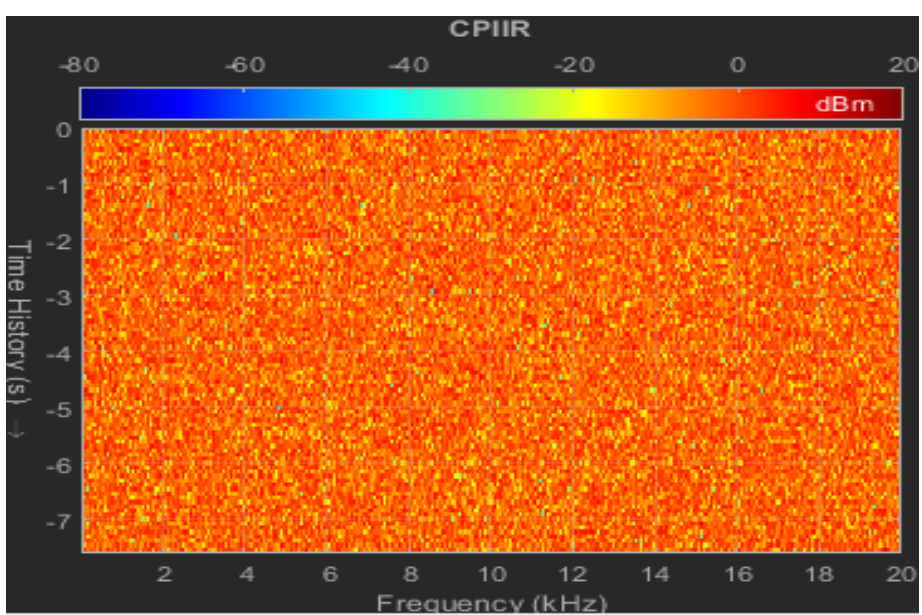

Fig. 6 Spectrogram (Power spectrum analysis)

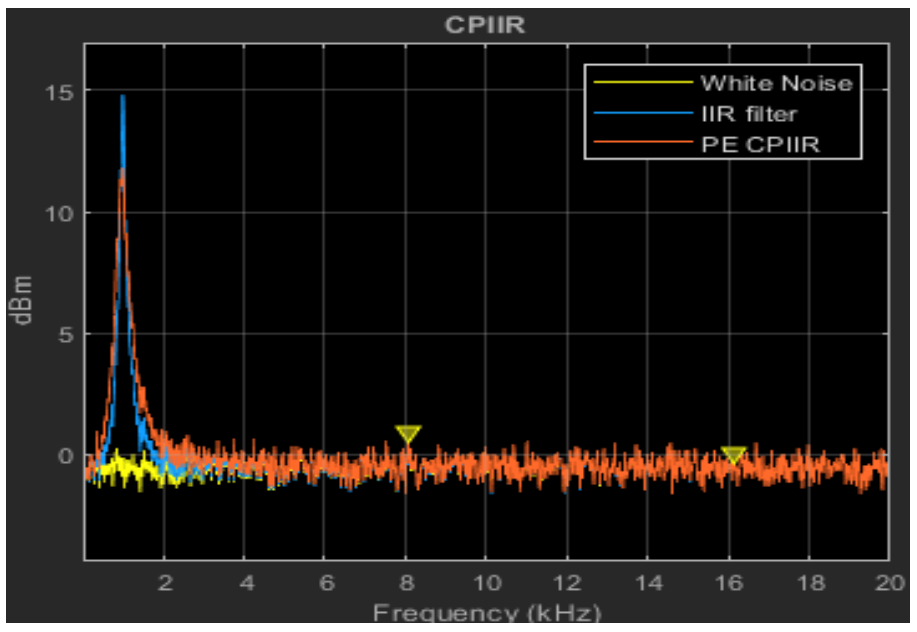

Fig. 7 Distortion measurement (8.11 KHz, 0.85 dB; $16.2 \mathrm{KHz},-0.91$ dB)

\section{CONCLUSION}

Considering the significance of a nonlinear real-time DSP filtering solution, in this paper a novel continuously programmable IIR digital filter is proposed. 


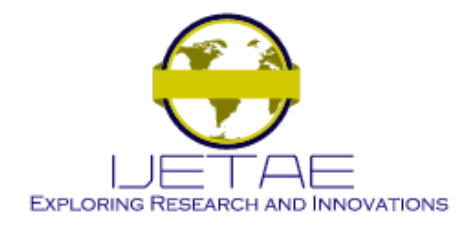

\section{International Journal of Emerging Technology and Advanced Engineering Website: www.ijetae.com (ISSN 2250-2459, ISO 9001:2008 Certified Journal, Volume 10, Issue 8, August 2020)}

Unlike classical solutions where authors have proposed ripple and/or square-error constrained meta-heuristic filtering concepts, in this paper an analytical approach based adaptive CPIIR model was developed. The proposed CPIIR model amalgamated parametric equalization concept with adaptive coefficient estimation method for non-linear real-time signal filtering. Dissimilar to the classical meta heuristic approaches which often undergoes local minima and convergence issues during optimal set of design parameter estimation, our proposed method applied mathematical concept where at fist equalization concept exploited the error information (difference between the expected signal and the observed filter response) to select the coefficient values dynamically. The proposed parametric equalization concept applied gain, frequency and bandwidth information which are the generic design specifications to assist coefficient estimator, which applied a simple mathematical calculation method for adaptive coefficient estimation and (design) tuning. This process continues iteratively till the filer achieves convergence or minimum error in output response. This approach at first reduced the computational complexity while retaining low power consumption, hardware demands and delay. Moreover, the proposed approach reduced the order of filter (here, merely $2^{\text {nd }}$ order direct filter) which can have higher significance towards energy, delay and memory consumption. Observing the proposed filter design and its corresponding outcome, especially magnitude response (Fig. 1), it can be found that the selection of Q-parameter is of vital response. Selection of high-value Q (say, 12) can force filter to show too narrow-band, while the small value of Q (say, 0.25) can cause too wideband response. Since, the proposed model intended to optimise global gain parameter in synch with the cut-off frequency and bandwidth, our focus was not to achieve higher pass-band. In future, the focus could be made on optimizing Q-factor to achieve targeted pass-band performance. Though, the proposed method achieved satisfactory performance, the concept of parametric equalization can be amalgamated with certain meta-heuristic with low computational overheads and iterations to enable overall better performance.

\section{REFERENCE}

[1] S.K. Mitra, Digital Signal Processing: A Computer-based Approach, 2nd ed., McGraw Hill, New York, 2001.

[2] J.G. Proakis, Digital Signal Processing: Principles, Algorithms, and Applications, Prentice Hall of India, New Delhi, 1997.

[3] B. Somanathan Nair, Digital Signal Processing: Theory, Analysis and Digitalfilter Design, Prentice-Hall of India, New Delhi, 2004.

[4] L. Tan, Digital Signal Processing: Fundamentals and Applications, Academic Press, New York, 2011.
[5] A. Hemdani,M.Wissem Naouar,I. S. Belkhodja,E. Monmasson,"Design of a Digital IIR Filter for Active Filtering Applications, "IEEE international conference in year 2012.

[6] S. K. Yadav, R. Mehra "Analysis of Different IIR Filter Based on Implementation Cost Performance" International Journal of Engineering and Advance Technology, Vol. 3, no. 4, pp. 267-270, April 2014.

[7] S. Moradi, Y. S. Kavian, "Hardware Description of Digital Adaptive IIR Filters for Implementing on FPGA", International Journal of Computer Applications, Vol. 65, no. 18, pp. 33-39, March 2013.

[8] S. kumari, A. Raghuvanshi, "RISC implementation of optimal programmable digital IIR filter", International Journal of Advanced Research in Electronics and Communication Engineering (IJARECE) Volume 4, Issue 2, February 2015, pp. 262-65.

[9] S. Roy, L. Srivani, D. T. Murthy, "Digital Filter Design Using FPGA", International Journal of Engineering and Innovative Technology (IJEIT) Volume 5, Issue 4, October 2015, pp. 78-82.

[10] [10] G. Vairetti, E. De Sena, M. Catrysse, H. Jensen, M. Moonen, and T. V. Waterschoot, "An automatic design procedure for loworder IIR parametric equalizers". AES: Journal of the Audio Engineering Society, 2018, vol. 66(11), pp. 935-952.

[11] J. B. Monteiro, A. Petraglia and C. A. Leme, "Capacitor charge control technique applied to digitally programmable IIR switchedcapacitor filter," Proceedings. 15th Symposium on Integrated Circuits and Systems Design, Porto Alegre, Brazil, 2002, pp. 68-73.

[12] J. B. Monteiro and A. Petraglia, "A 0.8/spl mu/m CMOS programmable IIR SC filter," 2004 IEEE International Symposium on Circuits and Systems (IEEE Cat. No.04CH37512), Vancouver, BC, 2004, pp. I-869.

[13] M. Omair Ahmad and Shenghong Wang, "A novel fully programmable switched-current IIR filter," Proceedings of CICC 97 - Custom Integrated Circuits Conference, Santa Clara, CA, USA, 1997, pp. 267-270.

[14] R. Hourani, H. Alassaly and W. Alexander, "Hardware implementation of IIR digital filters for programmable devices," 2013 IEEE 20th International Conference on Electronics, Circuits, and Systems (ICECS), Abu Dhabi, 2013, pp. 783-786.

[15] A. U. Madanayake and L. Bruton, "A high performance distributedparallel-processor architecture for 3D IIR digital filters," 2005 IEEE Intl. Symposium on Circuits and Systems, Kobe, 2005, pp. 14571460 Vol. 2

[16] F. O. Simons, A. D. George, B. A. Freathy and J. Medina, "Design and simulation of continuously programmable digital filter systems," Proceedings of 26th Southeastern Symposium on System Theory, Athens, OH, USA, 1994, pp. 255-259.

[17] V. A. Lesnikov, A. V. Chastikov, T. V. Naumovich and S. V. Armishev, "Implementation of a new paradigm in design of IIR digital filters," 2010 East-West Design \& Test Symposium, St. Petersburg, 2010, pp. 156-159.

[18] S. Ocloo and W. Edmonson, "An Interval-based Algorithm for Adaptive IIR Filters," 2006 Fortieth Asilomar Conference on Signals, Systems and Computers, Pacific Grove, CA, 2006, pp. 258262.

[19] M. A. Basiri M and N. M. Sk, "Configurable Folded IIR Filter Design," in IEEE Transactions on Circuits and Systems II: Express Briefs, vol. 62, no. 12, pp. 1144-1148, Dec. 2015.

[20] T. M. Jamel and K. Kais Naji, "New Performance Enhancement of Adaptive IIR Filtering Applications," 2018 Third Scientific Conference of Electrical Engineering (SCEE), Baghdad, Iraq, 2018, pp. 7-12. 


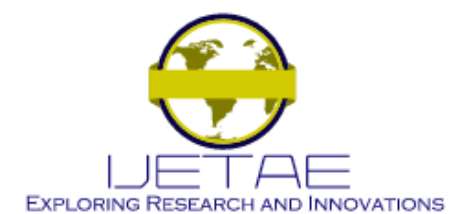

\section{International Journal of Emerging Technology and Advanced Engineering} Website: www.ijetae.com (ISSN 2250-2459, ISO 9001:2008 Certified Journal, Volume 10, Issue 8, August 2020)

[21] D. Dolgikh and P. Plotnikov, "Linear Polyphase Equalizer Based on Adaptive IIR Filter," 2019 International Multi-Conference on Engineering, Computer and Information Sciences (SIBIRCON), Novosibirsk, Russia, 2019, pp. 0026-0030.

[22] N. Agrawal, A. Kumar and V. Bajaj, "Optimized design of digital IIR filter using artificial bee colony algorithm," 2015 International Conf. on Signal Proc., Computing and Control, Waknaghat, 2015, pp. 316-321.

[23] N. Agrawal, A. Kumar, V. Bajaj and H. -. Lee, "Controlled ripplebased design of digital IIR filter," 2016 IEEE International Conference on Digital Signal Processing (DSP), Beijing, 2016, pp. 627-631.

[24] J. Liang and H. Keung Kwan, "IIR Filter Design Using Constrained Multi-Objective Cuckoo Search Algorithm," 2018 IEEE Canadian Conference on Electrical \& Computer Engineering, Quebec City, QC, 2018, pp. 1-4.

[25] F. Serbet, T. Kaya and M. T. Ozdemir, "Design of digital IIR filter using Particle Swarm Optimization," 2017 40th International Convention on Information and Communication Technology, Electronics and Microelectronics (MIPRO), Opatija, 2017, pp. 202204.

[26] R. Raju and H. Keung Kwan, "IIR Filter Design Using MultiObjective Artificial Bee Colony Algorithm," 2018 IEEE Canadian Conference on Electrical \& Computer Engineering, Quebec City, QC, 2018, pp. 1-4.

[27] S. K. Sarangi, R. Panda and A. Sarangi, "Design of adaptive IIR filter with modified firefly algorithm for parameter estimation," 2018 Technologies for Smart-City Energy Security and Power, Bhubaneswar, 2018, pp. 1-5.

[28] K. K. Dhaliwal and J. S. Dhillon, "On the design and optimization of digital IIR filter using oppositional artificial bee colony algorithm," 2016 IEEE Students' Conference on Electrical, Electronics and Computer Science (SCEECS), Bhopal, 2016, pp. 1-9.
[29] H. Aimi and K. Suyama, "Design of IIR filters with constraints using multi-swarm PSO," 2016 International Symposium on Intelligent Signal Processing and Communication Systems (ISPACS), Phuket, 2016, pp. 1-6.

[30] Zhenbin Gao, Xiangye Zeng, Jingyi Wang and Jianfei Liu, "FPGA implementation of adaptive IIR filters with particle swarm optimization algorithm," 2008 11th IEEE Singapore International Conference on Communication Systems, Guangzhou, 2008, pp. 1364-1367.

[31] T. Jinn-Tsong, C. Jyh-Horng, L. Tung-Kua, "Optimal design of digital IIR filters by using hybrid Taguchi genetic Algorithm”, IEEE T Ind Electron 2006; 53: pp. 867-879.

[32] U. Zolzer, Digital audio signal processing. John Wiley \& Sons, 2008.

[33] G. Ramos and J. J. Lopez, "Filter design method for loudspeaker equalization based on IIR parametric filters," J. Audio Eng. Soc, vol. 54, no. 12, pp. 1162-1178, 2006.

[34] H. Behrends, A. von dem Knesebeck, W. Bradinal, P. Neumann, and U. Zolzer, "Automatic equalization using parametric IIR filters," J. Audio Eng. Soc, vol. 59, no. 3, pp. 102-109, 2011.

[35] Z. Chen, Y. Liu, G. Geng, and F. Yin, "Optimal design of digital audio parametric equalizer," J. Inform. Comput. Sci., vol. 11, no. 1, pp. 57-66, 2014.

[36] P. Regalia and S. Mitra, "Tunable digital frequency response equalization filters," IEEE Trans. Acoust. Speech Signal Process., vol. 35 , no. 1, pp. 118-120, 1987.

[37] V. Valimaki and J. D. Reiss, "All about audio equalization: solutions and frontiers," Appl. Sciences, vol. 6, no. 5, p. 129, 2016.

[38] J.-M. Jot, "Proportional parametric equalizers application to digital reverberation and environmental audio processing," in Preprints Audio Eng. Soc. Conv. 139, 2015.

[39] G. Ramos and J. J. Lopez, IFilter design method for loudspeaker equalization based on IIR parametric filters," J. Audio Eng. Soc, vol. 54, no. 12, pp. $1162\{1178,2006$. 